(I) Besides albumen, fat, and carbohydrates, man requires for his nourishment certain substances which are contained in animal and vegetable food in varying quantities. These substances are both in quantity and quality related to the nitrogenous ingredients of food.

(2) Digestive and other ferments, the internal secretions, protective matters, \&c., are nitrogenous substances or descendants of such; their production must therefore be dependent to a certain extent on the intake of albumen and the conversion of the same in the body.

(3) Until the relations both of quantity and of quality are better understood, it will be advisable for general biological and hygienic reasons to favour an ample proportion of protein in practical nutrition, and not to limit the amount of protein to the minimum with which nitrogenous equilibrium can apparently be maintained.

The alcohol question also was to the fore, but a somewhat guarded opinion seemed to be expressed regarding its supposed evil effects when used in moderation. Dr. Triboulet, of Paris, considered that alcoholic drinks had an unfavourable action in cases of tuberculosis, and that alcohol diminishes resistance and predisposes to tuberculosis. Dr. Moeli, of Berlin, considered that alcohol is not a food, is not necessary, and in many cases is detrimental, and that its use should be discouraged in every way, not only by teaching the masses the evil effects of its use, but by instituting other interests so that the lack of it should not be felt.

Industrial diseases, particularly lead and mercurial poisoning, and those arising from dust, strain, \&c., were the subject of several papers, and many suggestions were made to mitigate these evils. Lighting, ventilation and water supplies were also dealt with, and likewise statistics. According to Prof. Ballod, the recent Prussian mortality tables show that as regards mortality the country has a decided superiority over the towns, but that this superiority is only distinctly noticeable among the male sex, and at ages $40-50$ years the towns appear to be slightly more favourable than the country.

At the general meeting telegrams of congratulation were received from the German Emperor and Empress, Lord Lister, and others, and three special lectures by English, French, and German men of science respectively were delivered. Dr. Haldane discussed his researches on the effects of high pressures and temperatures in underground workings on man. No ill effects result with pressures below about three atmospheres, but above these, unless the pressure is increased or decreased step by step, as the case may be, grave effects may be produced. As regards temperature, it is the wet-bulb temperature which determines the suitability of atmospheric conditions in mines. The optimum wet-bulb temperature is below $27^{\circ}$ C., the maximum that can be sustained without danger being $3 \mathrm{I}^{\circ} \mathrm{C}$. Prof. Chantemesse (Paris) discussed the serum treatment of typhoid fever. By growing the typhoid bacillus in a spleen broth medium for a week, heating to $55^{\circ} \mathrm{C}$., and injecting into horses, the serum of the latter acquires properties which usually act favourably on the course of the disease when injected into the patient. Thus, in the Paris hospitals from 190I-7, among 562 I cases the mortality was i 7 per cent., but during the same period in Iooo cases treated by Prof. Chantemesse with his serum the mortality was only 4.3 per cent. Prof. Schattenfroh, of Vienna, lectured on the hygienic care of water supplies, and the chemical and bacteriological examinations of drinking water. He urged that an international commission should be formed to devise standard methods for carrying out the latter.

The museum arranged in connection with the congress was of the greatest interest. The exhibits of the Imperial Board of Health and of the Institute for
Infectious Diseases were especially noteworthy. They consisted of cultivations of pathogenic micro-organisms and drawings and photographs of the same, patiological specimens of tuberculosis and other diseases, series of specimens demonstrating agglutination and precipitin tests for blood, travelling laboratories, \&c. All the principal Continental firms showed chemical and bacteriological apparatus, microscopes, and other instruments, and many of the casts showing pathological conditions were marvels of modelling. The exhibit of the Bacteriological Institute of Rio de Janeiro also was a large and comprehensive one, showing what good work is being done abroad, and it is to be regretted that exhibits from the British Empire seemed to be almost entirely wanting.

Demonstrations on the use of apparatus were given, and one interesting exhibit under the microscope was that of living active spirochætes, minute microorganisms which occur in relapsing fever, syphilis, and certain animal diseases.

\section{THE HARD AND SOFT STATES IN DUCTILE METALS.}

$W^{\text {THEN the early craftspen first observed that }}$ the metals they wry in were made harder by hammering, and that beriofinal softness could be again restored to the hardened metal by heat, it probably did pot to them that any explanation of thes userul phoperties was called for. At a later period, when an interest in the reasons for things became more general, it is probable that hardening was attrlbuted to the compacting of the substance by the driving of its particles closer together so that the mass as a whole became less open or porous. In the same way heat annealing was probably assumed to act by permanently expanding the metal and opening up its texture. So many analogies to these operations were ready to hand from the most common and everyday experiences that it is not surprising that even on closer inquiry this explanation should continue for a time to be accepted as sufficient, the more so as it was obviously true that in some cases unworked metal had an openness or porosity which could be removed by hammering or working. While the researches of chemists on the density of the metals showed plainly that increase of density does not alwavs result from compacting by pressure, these researches were probably too far removed from the ken of those who were most intimately concerned with the working of metals to arouse them to the insufficiency of the existing explanation of hardening.

In the latter part of the past century the views of physicists and chemists on the influence of molecular structure on the properties of matter began to find application in the field of scientific metallurgy. In particular, much attention was directed to the study of the crystalline constituents of alloys and to the influence of heat treatment on their equilibrium. The study of the iron alloys also led to the development of the view that iron itself can exist in several allotropic forms; thus the idea of allotropy was introduced into practical metallurgy.

Mr. G. T. Beilby's researches, ${ }^{1}$ which form the subject of the present article, indicate that all crystalline substances can also exist in a non-crystalline or amorphous form, and that the properties of these two forms are so distinct that they must be regarded as definite allotropic modifications. Observations on the stability of these forms, and on the conditions under which the one form can pass into the other, confirm this view. As these general principles have been found to apply

1 "The Hard and Soft States in Ductile Metals." By G. T. Beilby F.R.S. Paper read before the Royal Society on June 27. NO. I979, vOL. 76] 
without any exception over a wide range of suibstances, it appears justifiable to conclude that they are universally applicable. The subject is therefore a very extensive one, and the immediate researches which are dealt with here refer to only a small corner of a very wide field.

In the light of present knowledge it would now seem as if the phenomena of the hard and soft states are so striking that they might have been expected to stimulate inquiry into their true meaning at a much earlier date. One of the most obvious of these phenomena has been perfectly familiar ever since metals were first drawn into wire-that is, that the tenacity of the metal is enormously increased by the operation. By the simple operation of wire-drawing, the power of pure soft iron to resist stretching is raised from twenty tons per square inch to more than eighty tons. Recent researches with metals in the highest state of purity have shown that the resistance of gold to stretching may be raised from $4^{\frac{1}{2}}$ tons per square inch to more than It tons, while silver and copper are affected to an even greater extent.

Until very recently the adjective " crystalline," when applied to a metal, at once suggested hardness and brittleness, and even yet among practical metallurgists this association of jdeas is not easily got rid of. It is no paradox, however, to say that in the pure ductile metals the, crystalline state is actually the soft state. In what follows it will be shown that a very large part of this softness is directly due to the instability of the crystalline structure. Conversely, the non-crystalline or amorphous state is the more stable mechanically, and is therefore the harder. Not only the softness, but also the malleability and ductility of a metal, largely depend on its crystalline condition. When the metal is mechanically worked, as by hammering or rolling it into sheets or bars, or by drawing it through dies into rods or wires, some of the crystalline is broken down and passes into the non-crystalline form, and as the metal thereby becomes harder it is also reduced to a lower condition of malleability and ductility.

It has been concluded from a long series of experimental observations that in the passage from the crystalline to the non-crystalline state there is an intermediate stage during which the molecules have the freedom and mobility of the liquid state, and that the amorphous state results from the sudden congealing of this mobile phase. It is well known that when a substance passes from the liquid to the solid state, time is required for the molecules to marshal themselves in the orderly formation which is the essential feature of the crystalline state. If a liquid can be congealed with sufficient suddenness, the solid which results is non-crystalline or amorphous, e.g. glass, sugar-candy, \&c. If we were able to see the actual molecules we may imagine that the amorphous solid would present the appearance of an instantaneous photograph of a liquid in which the molecules would appear as if transfixed in the midst of their rapid movements. It follows from the above that if it were possible in a mass of metal simultaneously to break down all the crystalline units of structure with sufficient suddenness, the whole mass would for an instant be in the liquid condition, and on re-solidification would appear in the non-crystalline state. A little consideration, however, will make it plain that these conditions cannot be fulfilled in the ordinary mechanical operations on metals in the solid state. In a mass of metal, any stresses which are applied mechanically must be applied from, the outside, and can only reach an internal point or surface after passing through all the intervening layers. It follows that the breaking down and "flow" of the crystalline elements must take place step by step, so that the mobile condition occurs at successive surfaces within the mass. A wave of mobility can in certain cases be seen as it passes along a stressed rod, but it is instantly followed by a wave of congelation which leaves the metal behind it in a harder and more resistant condition. In some cases a second wave of mobility may be started by the application of a greater stress, but as a rule each successive application of a uniformly increasing stress produces less and less effect. The portions of metal which have yielded and flowed and again congealed protect those portions which still retain their crystalline structure. There appear to be good grounds for believing that even in a gclu leaf, in which the metal has been beaten to a thickness of only $\mathrm{I} / 280,000$ of an inch, there are still minute units in the crystalline state which have escaned destruction owing to the protective action of the harder, non-crystalline metal in which they are embedded. Gold wires which have been drawn through a wire plate until they are fifteen times their original length show a microstructure in which deformed and broken down crystals are embedded in non-crystalline substance. The hardened metal is a complex structure built up of crystalline and noncrystalline substance; in studying its properties, therefore, it is necessary to remember that no specimen, however drastic may have been its mechanical treatment, can be entirely in the non-crystalline condition.

Though an increase of hardness and tenacity is a very conspicuous feature of the change from the one state to the other, it is only one among a number of equally definite indications of change. A comparison of the heat of solution of a metal in the two states shows that the molecular energy stored in the noncrystalline form is greater than in the crystalline. In this case the difference in solubility which results from this greater energy is further accentuated as the two phases of the metal act towards the solvent as a galvanic couple. A thermoelectric couple made by twisting together the ends of wires in the hard and soft condition is affected by changes of temperature in the same way as a couple made of two different metals would be. In the case of silver a thermocouple of this description can develop an e.m.f. of 27 microvolts for a temperature difference of $83^{\circ}$. In all these cases the single chemically-pure metal behaves like two distinct metals.

When hardened metal is heated to a certain temperature, its softness is completely restored. The microscope shows that when this occurs complete crystalline rearrangement has also taken place. The micrographs, Figs. I and 2, from the paper by $\mathrm{Mr}$. Beilby read before the Royal Society on June 27, show the two types of structure, the hard and the soft. In Fig. I the original crystalline grains have been completely broken down and destroyed by wiredrawing, giving place to masses of deformed and shattered crystal units cemented or concreted together by that part of the metal which has flowed and congealed. In Fig. 2 a new crystalline structure has been developed by heat, and all traces of the other structure have disappeared. This re-crystallisation in hardened metal occurs at a temperature far below the melting point of the metal. In gold the re-crystallisation temperature is about $280^{\circ}$, while its melting point is $1080^{\circ}$; this profound change of structure, therefore, occurs $800^{\circ}$ below the melting point.

In the crystalline state the molecules are disposed in sheets or lamellæ of uniform orientation, like soldiers in a battaiion. In the liquid state the molecules are in free movement; they do not maintain fixed positions with respect to each other. The effect of sudden con-

No. I 979, vOL. 76] 
gealing probably is to bind the molecules into a rigid mass quite irrespective of any special polar attractions which they may have for each other. They are bound together by the general cohesive force, and their freedom of movement is much curtailed. This is evident not only because they cannot move sufficiently freely to take up the regularly oriented arrangement, but

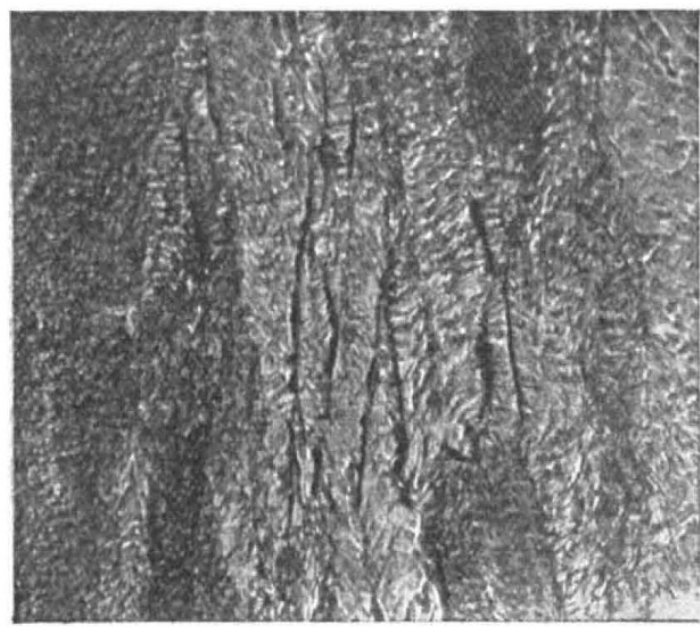

FIG. I. - Hard-drawn gold wire. Magnification $\times 700$.

also because their elasticity in this state is much less perfect than it is in the crystallised state; the molecules cannot vibrate freely in the amorphous state.

The effect of raising the temperature to the crystallising point is to raise the kinetic energy of the molecules, and therefore to neutralise a part of the cohesive force, thus weakening it and enabling the

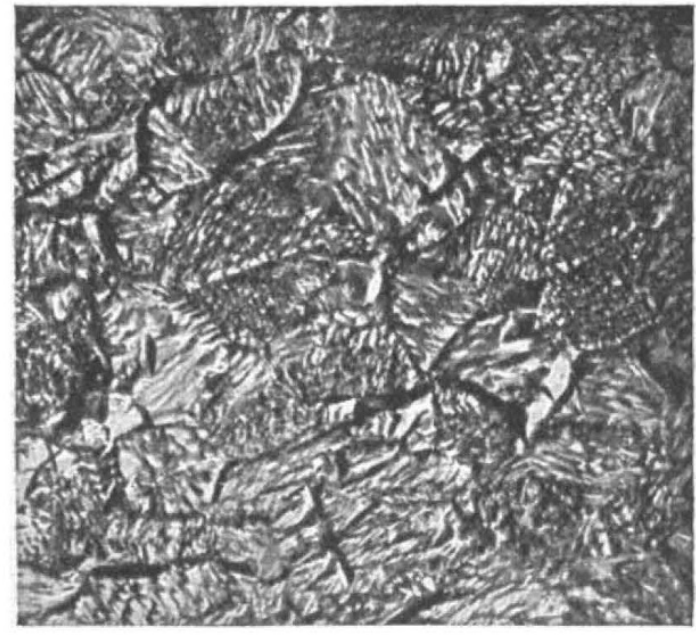

FrG. 2.-Hard-drawn gold wire after crystallisation at $280^{\circ}$. Magnification $\times 700$.

molecules to spring into their uniformly oriented position; the crystalline state is thus restored and the internal energy of the mass is reduced. The present observations show that crystallisation occurs over a short and definite range of temperature-short, that is to say, compared with the ranges above and below the crystallisation range. Below the crystallisation range the amorphous or non-crystalline form of the metal is known by direct observations to be perfectly stable down to $-180^{\circ}$, while above that range the crystalline form is stable up to the temperature of liquefaction.

The changes in the electrical, mechanical, and other properties, which occur when the crystallisation range is reached, amply confirm the microscopic observations, and all point to the occurrence of an important change in the molecular structure.

By means of an acoustical method it has been found possible to detect the minute changes in elasticity which occur as the temperature is gradually raised to the crystallising range. In this way it has been made evident that there are two distinct stages in the relief of the molecular strains which are caused by hardening. In the first stage no important alteration in the other properties of the hardened metal occurs, while in the second and more important stage the complete restoration of elasticity exactly corresponds with the other changes which occur at the crystallisation temperature.

\section{OCTOBER METEORS.}

CTOBER is a month when metoy/s are decidedly numerous. They are refticylarly abundant from October ${ }^{15}$ to Octobs ${ }_{2}$, and this period includes the well-km nower of Orionids, but the conditions in be $\mathcal{}$ undavourable this year owing to the fufl moor of October 21 .

Early in tife month there is occasionally a rich show near the northern boundary of Boötes at $230^{\circ}+52^{\circ}$, and on October 8 there are many meteors from Aries about $42^{\circ}+20^{\circ}$, from Auriga, $77^{\circ}+32^{\circ}$, and from about this date to October 20 there is a well pronounced morning shower of long-pathed meteors from $154^{\circ}+39^{\circ}$.

Before sunrise the observer will also trace radiants at $101^{\circ}+1^{\circ}, 100^{\circ}+13^{\circ}, 108^{\circ}+13^{\circ}, 121^{\circ}+0^{\circ}, 133^{\circ}+68^{\circ}$, $133^{\circ}+48^{\circ}$.

Thirty years ago, viz. in 1877 , during the first week in October there were very well pronounced showers at $133^{\circ}+79^{\circ}$ and $313^{\circ}+77^{\circ}$; the former gave swift streak-leaving meteors, the latter slow faint meteors.

October furnishes several radiants of Perseids, and one of the most notable of these agrees precisely in its apparent position with the centre of the great display of August Perseids on the date of maximum. Between October 8 and I4 I have recorded a number of slow meteors from a well defined radiant at $45^{\circ}+5^{\circ}$.

The principal shower in the last half of October is one of Arietids from near $\epsilon$. On 1877 October 28November I, I saw thirty-one meteors from $43^{\circ}+22^{\circ}$, and on $\mathrm{I} 887$ October $\mathrm{II}-24$, forty-five meteors were registered from $40^{\circ}+20^{\circ}$. The members of this system are white, rather slow, and occasionally brilliant, with trains of yellow sparks. As they were very active in 1877 and 1887 , they may periodically recur at intervals of a decade, and be numerously manifested again in 1907 .

W. F. Denning.

MR. HALDANE ON SCIENCE IN COMMERCE.

A VERBATIM report of the speech on scientific A commercial education in relation to the successful pursuit of trole, delivered by $\mathrm{Mr}$. Haldane at Liverpool on Sep (ember 19, and briefly mentioned in last week's NAT RE, appeared in the Liverpool Daily Post of Septensh 20 . The two main points developed by Mr. Haldane in the course of his remarks, were the domination of mifhd over matter and the value of 